\title{
Mass and angular momentum loss of first stars via decretion disks
}

\author{
Jiří Krtička ${ }^{1}$, Stanley P. Owocki ${ }^{2}$ and Georges Meynet ${ }^{3}$ \\ ${ }^{1}$ Department of Theoretical Physics and Astrophysics, Masaryk University, CZ-611 37 Brno, \\ Czech Republic \\ ${ }^{2}$ Bartol Research Institute, University of Delaware, Newark, DE 19716, USA \\ ${ }^{3}$ Geneva Observatory, CH-1290 Sauverny, Switzerland
}

\begin{abstract}
Although the first stars were likely very hot and luminous, their low or zero metallicity implies that any mass loss through winds driven by line-scattering of radiation in metal ions was likely small or non-existent. Here we examine the potential role of another possible mechanism for mass loss in these first stars, namely via decretion disks associated with near-critical rotation induced from evolution of the stellar interior. In this case the mass loss is set by the angular momentum needed to keep the stellar rotation at or below the critical rate. In present evolutionary models, that mass loss is estimated by assuming effective release from a spherical shell at the surface. Here we examine the potentially important role of viscous coupling of the decretion disk in outward angular momentum transport, emphasizing that the specific angular momentum at the outer edge of the disk can be much larger than at the stellar surface. The net result is that, for a given stellar interior angular momentum excess, the mass loss required from a decretion disk can be significantly less than invoked in previous models assuming a direct, near-surface release.
\end{abstract}

Keywords. Stars: mass-loss - stars: evolution - stars: rotation

\section{Mass-loss from first stars}

The first stars were pure hydrogen-helium. Numerical simulations of their formation show they were very massive and luminous. Current-day massive stars lose mass via strong winds driven by line-scattering from metal ions, but the lack of metals in the first stars implies any line-driven wind would be weak or non-existent (Krtička \& Kubát 2006).

Nonetheless, there are still processes that can lead to the mass-loss from first stars. In particular, their rotational evolution can bring them close to the critical rotation limit (Meynet et al. 2006), allowing a mechanical ejection of mass via an equatorial "decretion" disc. Here we examine the potential role of this mass loss mechanism for first stars.

\section{Stellar outflows via winds vs. decretion disks}

There are some key difference between winds and decretion disks. In winds the mass loss rate sets the angular momentum loss, whereas in disks the angular momentum loss needed to keep the stellar rotation subcritical sets the mass loss rate.

Rotating stars at very low metallicity are expected to reach critical rotational velocity more easily because they lose less mass and angular momentum by line-driven stellar winds.

At the critical limit, the instantaneous mass losses depends on the extension of the decretion disk. In the disk, angular momentum is transported outward by viscous effects. 


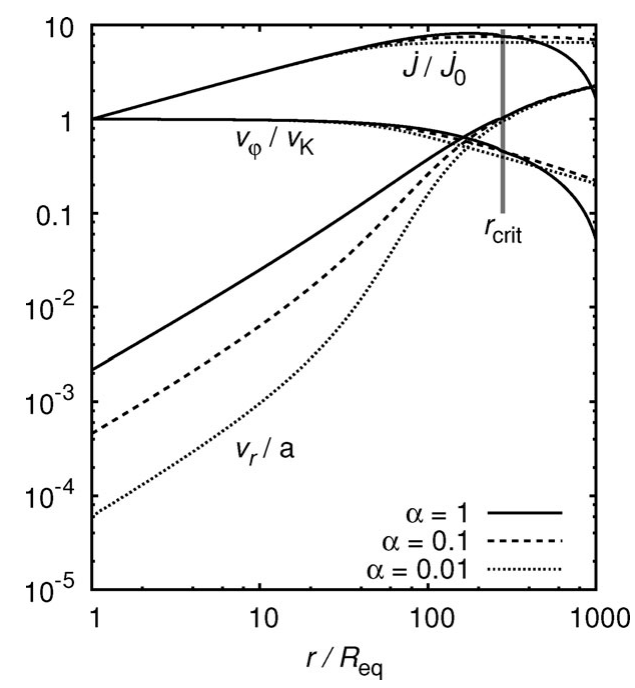

Figure 1. The dependence of the radial (in units of the sound speed $a$ ) and longitudinal (in units of the Keplerian velocity $v_{\mathrm{K}}$ ) velocities, and the angular momentum loss in units of the equatorial angular momentum loss $\dot{J}_{0}$ on the radius in a viscous disk. The results are plotted for different viscosity paremeters $\alpha$ of Shakura \& Sunyaev (1973).

Its extension is fixed at the point where matter decouples, keeping its angular momentum constant. Fig. 1 shows that for a thermal expansion of an isothermal disk the typical disk extension is a few hundred times the stellar equatorial radius.

The mass loss rates needed to maintain the star just below the critical limit is thus much lower when a decretion disk is formed than in previous models that assume direct loss from the stellar surface. In the example of Fig. 1, one infers that the disk reduces the mass loss rate by a factor ten or more.

We provide prescriptions for computing the instantaneous mass loss rates in the presence of a decretion disk and for different physical circumstances:

case A: very short radiative ablation timescale of the disk

case B: disk extension is by viscosity and thermal expansion alone

case C: both viscosity and radiative effects determine the extension of the disk.

In the first stars, case B is the most likely one.

\section{Acknowledgement}

This work was supported by grant GA ČR 205/07/0031. SPO acknowledges support of NSF grant AST-0507581 and NASA grant NNG05GC36.

\section{References}

Krtička, J. \& Kubát, J. 2006, A\&\&A, 446, 1039

Meynet, G., Ekström, S., \& Maeder, A. 2006, A\&A, 447, 623

Shakura, N. I. \& Sunyaev, R. A. 1973, A\&AA, 24, 337 